\title{
The Distribution of Opposite Sugeno Integral of Uniformly Distributed Vector Is Uniform ${ }^{\dagger}$
}

\author{
Haruki IMAOKA*
}

\begin{abstract}
We introduce a new fuzzy integral named opposite Sugeno integral. It can be considered to be an averaging operator because it has three essential properties of an averaging operator such as monotonicity, continuity and value between min and max. Usually an averaging operator shrinks variance. It is, however, proved that if fuzzy measure is assumed to be additive and the probability distribution of input vector is assumed to be uniform, the probability distribution after opposite Sugeno integral is operated is also uniform.
\end{abstract}

Keywords : Fuzzy Integral, Opposite Sugeno Integral. Uniform Distribution

\section{Introduction}

Fuzzy measure and fuzzy integral were originally defined by Sugeno[2]. The fuzzy integral has been called Sugeno Integral. Later, another fuzzy integral named opposite Sugeno integral was proposed, which has a property opposed to Sugeno integral[1]. The two fuzzy integral models are essentially based on the fuzzy measure and these definitions will be given in chapter 2 .

Here we want to clarify the properties that are required for an averaging operator. First of all, it is a real function of $n$-variables. Domain and range are assumed to be a unit $n$-cube and a unit closed interval. More precisely, it is a function of $n$ variables from $[0,1]^{n}$ to $[0,1]$. It must be a monotone function. It must be a continuous function. The function value must be between minimum and maximum of variable values. These three properties are essential terms to be an averaging operator.

To discuss averaging operators, incidentally, it is important to clarify whether there exist some parameters or not. The parameters of fuzzy integrals are coefficients of fuzzy measure.

Fuzzy integrals are indeed one of the classes of averaging operators and have the same parameters. For short, hereafter we sometimes call values of $n$-variables as integrand or input vector and the parameters as fuzzy measure respectively.

Fuzzy integral is a monotone and continuous function with respect to integrand. If integrand is logical, which means all values of $n$-variables are limited to be 1 or 0 , the integrated value coincides with corresponding coefficient of fuzzy measure

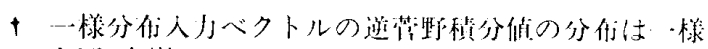
今湔 侸樹

* Faculty of Human Life and Environment, Nara Women's University

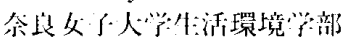

regardless of the model of fuzzy integrals. In other words, the values of the function of many variables on the vertices of the hypercube give the coefficients of the fuzzy measure.

Similarly it can be regarded as a monotone and continuous function w.r.t. fuzzy measure. If fuzzy measure is logical, which means all coefficients of fuzzy measure are limited to be 1 or 0 , the integrated values are the same regardless of the model. By using the monotonicity w.r.t. fuzzy measure, we can easily prove the property of value between maximum and minimum.

In chapter 3 , we focus on probability distribution or variance of the integrated values. To simplify the discussion, the probability distribution of input vector is limited to be the uniform distribution on the product space. Usually the variance after ordinary averaging is smaller than the original one, that is the variance of identical marginal distribution for each variable. The fact is explained by the central limit theorem. We want to call the fact a variance diminishing property. Averaging by opposite Sugeno integral, however, has the weakest variance diminishing property. If fuzzy measure is assumed to be additive measure, the probability distribution after opposite Sugeno integral is operated is also uniform. If fuzzy measure is not additive, the expression is not true. This is the main theorem in this paper.

\section{Preliminary}

In this paper, we limit our discussion of fuzzy integrals in finite case.

\subsection{Fuzzy measure space}

Definition 1: A triplet $(X, \wp(X), \mu)$ is said to be a fuzzy measure space, where $\wp(X)$ is a power set of $X$ and $\mu$ is a fuzzy measure, that is a set function from $\wp(X)$ to $[0,1], \mu$ is said to be a 
fuzzy measure if it has the following properties:

$$
\begin{aligned}
& \mu(\emptyset)=0, \mu(X)=1 . \\
& A \subseteq B \Rightarrow \mu(A) \leq \mu(B) .
\end{aligned}
$$

Fuzzy measure is an extension of the ordinary measure. If fuzzy measure is additive, it is reduced to the ordinary measure. The additivity is defined by

$$
A \cap B=\emptyset \Rightarrow \mu(A \cup B)=\mu(A)+\mu(B) .
$$

\subsection{Measurable function and its distribution func-} tion

Definition 2: A function $f$ from $X$ to $[0,1]$ is said to be measurable if

$$
\{x: f(x)>\alpha\} \in \wp(X), \quad \forall \alpha \in[0,1] .
$$

In finite case, every function is automatically measurable, and this fact guarantees the next definition.

Definition 3: Here we can define a distribution function of a measurable function $f$. The function $G$ from $[0,1]$ to $[0,1]$ is defined by

$$
G(\alpha):=\mu(\{x: f(x)>\alpha\}) .
$$

The function $G$ is a monotone decreasing simple function because the fuzzy measure $\mu$ is monotone increasing.

\subsection{Two fuzzy integrals in finite case}

It is assumed that a measurable function $f$ is a simple function determined by $a_{1} \leq a_{2} \leq \cdots \leq a_{n}$, where $a_{i}:=f\left(x_{i j}\right)$ is the $i$-th value of the sorted list. Let $\mu_{i}$ be $\mu\left(\left\{x_{(i)}, x_{(i+1)}, \ldots, x_{(n)}\right\}\right)$. Then the distribution function $G$ becomes a monotone decreasing simple function determined by $a_{1} \leq a_{2} \leq \cdots \leq a_{n}$ and $1=\mu_{1} \geq \mu_{2} \geq \cdots \geq \mu_{n} \geq \mu_{n+1}=0$. For short, hereafter we sometimes call them as an ordered list of integrand and an ordered list of fuzzy measure respectively. If there are some equalities in an ordered list of integrand, the ordered list of fuzzy measure can not be determined uniquely, but the distribution function can be determined uniquely.

Definition 4: Fuzzy integral is a functional of a distribution function. The two fuzzy integrals are defined by

$$
\text { (o) } \int f d \mu:=\sum_{i=1}^{n}\left\{\left(a_{i} \mu_{i}\right)-\left(a_{i} \mu_{i+1}\right)\right\}
$$

For Sugeno integral and opposite Sugeno integral, the notation $x \bigcirc y$ is replaced by $x \wedge y$ and $x \odot$ $y:=(x+y-1) \vee 0$ respectively.

\subsection{Notation for additive masure}

When fuzzy measure is assumed to be an additive measure, necessary coefficients are $\tau_{1}, \tau_{2}, \ldots$, $\tau_{n}$ which are defined by $\tau_{i}:=\mu\left(\left\{x_{i}\right\}\right)$.
Define $\sigma_{i}=\tau_{1}+\tau_{2}+\cdots+\tau_{i}$ and $\sigma_{0}=0$. If $n$-variables list is an ordered list i.e. $f\left(x_{1}\right) \leq f\left(x_{2}\right) \leq$ $\cdots \leq f\left(x_{n}\right)$, the ordered list of fuzzy measure is $1=$ $\sigma_{n} \geq \sigma_{n}-\sigma_{1} \geq \cdots \geq \sigma_{n}-\sigma_{n-1} \geq \sigma_{n}-\sigma_{n}=0$.

Here we want to express opposite Sugeno integral by using the above notation of additive measure. Note that $\sigma_{n}=1$. Define $y_{i}:=f\left(x_{i}\right)$ and assume they make an ordered list of integrand.

$$
\begin{aligned}
(\odot) \int f d \mu= & \sum_{i}^{n}\left\{\left(\left(y_{i}+1-\sigma_{i-1}-1\right) \vee 0\right)\right. \\
& \left.-\left(\left(y_{i}+1-\sigma_{i}-1\right) \vee 0\right)\right\} \\
= & \left.\sum_{i}^{n}\left\{\left(y_{i}-\sigma_{i-1}\right) \vee 0\right) \wedge \tau_{i}\right\} .
\end{aligned}
$$

The equality is easily shown.

\section{Non variance diminishing property of opposite Sugeno integral}

In this chapter, our concern is on a probability distribution function of integrated values. To simplify the discussion, we assume that the original distribution of input vector is the uniform distribution on the hypercube.

Definition 5: Assume that the integrand $f$ is a simple function represented by a vector $\left(y_{1}, y_{2}, \ldots\right.$, $\left.y_{n}\right)$. The cumulative distribution function $F(\beta)$ is defined by

$$
\begin{aligned}
F(\beta): & =P\left(\left(y_{1}, y_{2}, \ldots, y_{n}\right) \in[0,1]^{n}:() \int f d \mu \leq \beta\right) \\
& =\int_{0}^{1} d y_{n} \int_{0}^{1} d y_{n-1} \cdots \int_{0}^{1} u\left(\beta-() \int f d \mu\right) d y_{1},
\end{aligned}
$$

where $u(x)$ is a unit function defined by $u(x)=0$ if $x<0,1$ if $x \geq 0$.

Note that the order of integrals is arbitrary by Fubini's Theorem.

\subsection{In the case of two variables}

Here we discuss ordinary mean $m$ and variance $\sigma^{2}$ of the distribution function in the case of two variables. In this case, there are two coefficients of fuzzy measure and we denote them by $\tau_{1}$ and $\tau_{2}$. Let $z\left(y_{1}, y_{2}\right)$ be $(0) \int f d \mu$. By using the relations $m=\int_{0}^{1} d y_{2} \int_{0}^{1} z d y_{1}$ and $\sigma^{2}=\int_{0}^{1} d y_{2} \int_{0}^{1} z^{2} d y_{1}-m^{2}$, they are summarized as follows:

Sugeno integral :

$$
\begin{aligned}
m= & \frac{1}{3}+\frac{1}{6} \tau_{1}^{2}\left(3-2 \tau_{1}\right)+\frac{1}{6} \tau_{2}^{2}\left(3-2 \tau_{2}\right) \\
= & \frac{1}{2}+\frac{1}{12}\left(\tau_{1}+\tau_{2}-1\right)\left\{3-\left(\tau_{1}+\tau_{2}-1\right)^{2}\right. \\
& \left.-3\left(\tau_{1}-\tau_{2}\right)^{2}\right\}, \quad 1 / 3 \leq m \leq 2 / 3
\end{aligned}
$$




$$
\begin{aligned}
\sigma^{2}= & \frac{1}{12}-\left(m-\frac{1}{2}\right)^{2}-\frac{1}{2}\left\{\tau_{1}^{2}\left(1-\tau_{1}\right)^{2}+\tau_{2}^{2}\left(1-\tau_{2}\right)^{2}\right\}, \\
& 1 / 48 \leq \sigma^{2} \leq 1 / 12 .
\end{aligned}
$$

Opposite Sugeno integral :

$$
\begin{aligned}
m= & \frac{1}{2}+\frac{1}{12}\left(\tau_{1}+\tau_{2}-1\right)\left\{3-\left(\tau_{1}+\tau_{2}-1\right)^{2}\right. \\
& \left.-3\left(\tau_{1}-\tau_{2}\right)^{2}\right\}, \quad 1 / 3 \leq m \leq 2 / 3 . \\
\sigma^{2}= & \frac{1}{12}-\left(m-\frac{1}{2}\right)^{2}, \quad 1 / 18 \leq \sigma^{2} \leq 1 / 12 .
\end{aligned}
$$

In both fuzzy integrals, the variance did not exceed the value $1 / 12$ which is the variance of the original uniform distribution on the unit interval. This is an example of the variance diminishing property. In Sugeno integral, the variance $1 / 12$ appeared when fuzzy measure is both additive and logical. On the other hand, in opposite Sugeno integral, the variance $1 / 12$ appeared when fuzzy measure is only additive.

Now our concern is changed to the distribution function itself when fuzzy measure is additive.

Example: From the additivity, we can denote two coefficients of fuzzy measure by $\tau$ and $1-\tau$. The distribution function after opposite Sugeno integral is operated is expressed by

$$
F(\beta)=0 \text { if } \beta<0, \beta \text { if } 0 \leq \beta<1,1 \text { if } 1 \leq \beta \text {. }
$$

Proof: Here we can assume that $\tau \geq 1 / 2$ without losing generality.

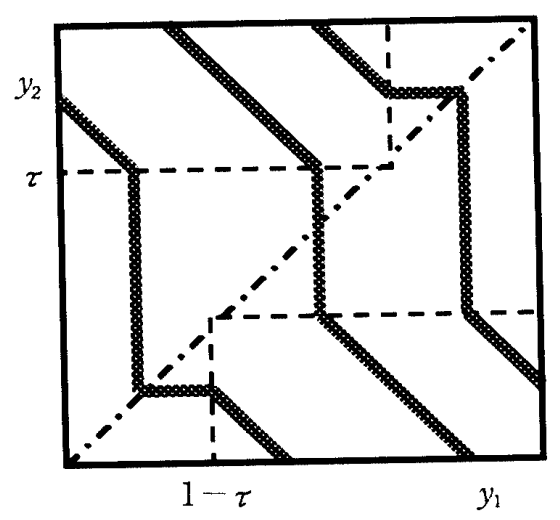

Fig.1 Contour plot of $z\left(y_{1}, y_{2}\right)$.

When $0 \leq \beta \leq 1-\tau$,

$$
\begin{aligned}
F(\beta)= & \int_{0}^{\beta}\left(\tau+\beta-y_{1}\right) d y_{1}+\int_{\beta}^{1-\tau} \beta d y_{1} \\
& +\int_{1-\tau}^{1-\tau+\beta}\left(1-\tau+\beta-y_{1}\right) d y_{1} \\
= & \beta .
\end{aligned}
$$

When $1-\tau<\beta \leq \tau$,

$$
\begin{aligned}
F(\beta)= & \int_{0}^{\beta-1+\tau} d y_{1}^{\prime}+\int_{\beta-1+\tau}^{\beta}\left(\tau+\beta-y_{1}\right) d y_{1} \\
& +\int_{\beta}^{1-\tau+\beta}\left(1-\tau+\beta-y_{1}\right) d y_{1} \\
= & \beta .
\end{aligned}
$$

When $\tau<\beta \leq 1$,

$$
\begin{aligned}
F(\beta)= & \int_{0}^{\tau-1+\beta} d y_{1}+\int_{\tau-1+\beta}^{\tau}\left(\tau+\beta-y_{1}\right) d y_{1} \\
& +\int_{\tau}^{\beta} \beta d y_{1}+\int_{\beta}^{1}\left(1-\tau+\beta-y_{1}\right) d y_{1} \\
= & \beta .
\end{aligned}
$$

As for $\beta \notin[0,1]$, it is obvious. []

\subsection{Non variance diminishing property of opposite Sugeno integral}

Theorem 1: We assume that the original probability distribution of each variable is the identical uniform distribution independently. For every additive mea sure, the probability distribution of integrated values by opposite Sugeno integral is also uniform.

In this section, we will prove the theorem. The theorem is very simple, but unfortunately the proof is very long.

\subsubsection{Notation and some simplifications}

First of all, we use $x_{i}$ instead of $y_{i}$ which was function value as mentioned before. As for fuzzy measure, we use $\tau_{i}$ and $\sigma_{i}$, which are not changed.

Here we define two functions $u(x)$ and $v(x)$ by

$$
u(x):=0 \quad \text { if } x<0,1 \text { if } x \geq 0,
$$

and

$$
v(x):=0 \text { if } x<0, x \text { if } 0 \leq x<1,1 \text { if } 1 \leq x .
$$

$u(x)$ is called a unit function, and $v(x)$ is the integrated function of $u(x)-u(x-1)$.

Proposition: Let $P_{n}$ be the set of permutations on $\{1,2, \ldots, n\}$. The distribution function is expressed by

$$
\begin{aligned}
F(\beta)= & \sum_{\pi \in P_{n}} \int_{0}^{\sigma_{n}} d x_{n} \int_{0}^{x_{n}} d x_{n-1} \cdots \\
& \int_{0}^{x_{2}} u\left(\beta-\sum_{i=1}^{n} \tau_{\pi(i)} v\left(\frac{x_{i}-\sigma_{i-1, \pi}}{\tau_{\pi(i)}}\right)\right) d x_{1},
\end{aligned}
$$

where $\sigma_{i-1, \pi}=\tau_{\pi(1)}+\tau_{\pi(2)}+\cdots+\tau_{\pi(i-1)}$. Here it is assumed that if $\tau_{i}=0$ then $\tau_{i} v\left(\left(x_{i}-\sigma_{i-1}\right) / \tau_{i}\right)=0$.

Proof: If $x_{1} \leq x_{2} \leq \cdots \leq x_{n}$, opposite Sugeno integral is expressed by $\left.\sum_{i=1}^{n}\left\{\left(x_{i}-\sigma_{i-1}\right) \vee 0\right) \wedge \tau_{i}\right\}$, and it coincides with $\sum_{i=1}^{n} \tau_{i} v\left(\left(x_{i}-\sigma_{i-1}\right) / \tau_{i}\right)$.

The integral covers a part of the domain which satisfy the order of $x_{i}$, Sum of every permutation of the ordered list of integrand covers all of the domain. Since fuzzy measure is additive, sum of every permutation of the ordered list can be expressed by sum of every permutation of $\tau_{i}$. 
Now the equation to be proved is

$F(\beta)=v(\beta)$.

Here we want to stress that $\sigma_{n}$ is used, instead of 1 , in the above expression of $F(\beta)$. From now on, the condition $\sigma_{n}=1$ is removed. Thus our target is that if $\sigma_{n}=1$ then $F(\beta)=v(\beta)$. It is easily shown that $F(\infty)=\left(\sigma_{n}\right)^{n}$, and this will be used in the proof.

Instead of proving the target equation directly, we use Laplace transform. By operating $\int_{0}^{\infty} e^{-s^{\beta}}$ $d \beta$, the left side of the equation is changed to

$$
\begin{aligned}
V(s):= & \int_{0}^{\infty} e^{-s \beta} F(\beta) d \beta \\
= & \frac{1}{S_{\pi \in P_{n}}} \int_{0}^{\sigma_{n}} e^{-s \tau_{\pi n n} v}\left(\frac{x_{n}-\sigma_{n-1, \pi}}{\tau_{\pi(n)}}\right) d x_{n} \\
& \int_{0}^{x_{n}} e^{-s \tau_{\pi n-1, v} v}\left(\frac{x_{n-1}-\sigma_{n-2, \pi}}{\tau_{\pi(n-1)}}\right) d x_{n-1} \\
& \cdots \int_{0}^{x_{2}} e^{-s \tau_{\pi(1)} v}\left(\frac{x_{1}-\sigma_{0}}{\tau_{\pi(1)}}\right) d x_{1},
\end{aligned}
$$

and the right side is changed to

$$
\frac{1-e^{-s}}{s^{2}}
$$

Here we define functions $V_{i}(t)$ and coefficients $V_{i}$ by

$$
\begin{aligned}
V_{i}(t):= & \int_{0}^{t} e^{-s \tau_{i} v}\left(\frac{x_{i}-\sigma_{i-1}}{\tau_{i}}\right) d x_{i} \cdots \\
& \int_{0}^{x_{2}} e^{-s \tau_{1} v}\left(\frac{x_{1}-\sigma_{0}}{\tau_{1}}\right) d x_{1}, \\
& V_{0}(t)=1
\end{aligned}
$$

and

$$
V_{i}:=V_{i}\left(\sigma_{i}\right) .
$$

When a permutation $\pi$ is applied to $\tau$, they are denoted by $V_{i, \pi}(t)$ and $V_{i, \pi}$.

Thus our target to be proved is that if $\sigma_{n}=1$ then

$$
\begin{aligned}
& V(s)=\frac{1}{S_{\pi}} \sum_{P_{n}} V_{n, \pi}=\frac{1}{s^{2}}\left(1-e^{-s}\right) \text { i.e. } \\
& \sum_{\pi \in P_{n}} V_{n, \pi}=\frac{1}{S}\left(1-e^{-s}\right),
\end{aligned}
$$

and we will prove it in more general form written by

$$
\sum_{\pi \in P_{n}} V_{n, \pi}=\frac{1}{S}\left(\sigma_{n}\right)^{n-1}\left(1-e^{-s \sigma_{n}}\right) .
$$

Note that the functions $V_{i}(t)$ are expressed by the following recursion equations:

$$
V_{i}(t)=\int_{0}^{t} e^{-s \tau_{i} v\left(\frac{x-\sigma_{i-1}}{\tau_{i}}\right)} V_{i-1}(x) d x .
$$

\subsubsection{Preparation}

Here we define two functions $h$ and $g$ which will appear in the proof by

$$
\begin{aligned}
& h_{p}^{r}(x):=\int_{\sigma_{p}}^{x} d t_{r} \int_{\sigma_{p-1}}^{t_{r}} d t_{r-1} \cdots \int_{\sigma_{p-r+1}}^{t_{2}} d t_{1}, \\
& h_{p}^{0}(x):=1,
\end{aligned}
$$

and

$$
\begin{aligned}
& g_{p}^{r}(x):=\int_{x}^{\sigma_{p+1}} d t_{r} \int_{t r}^{\sigma_{p+2}} d t_{r-1} \cdots \int_{t_{2}}^{\sigma_{p+r}} d t_{1}, \\
& g_{p}^{0}(x):=1 .
\end{aligned}
$$

When a permutation $\pi$ is applied to $\tau$, they are denoted by $h_{p, \pi}^{r}(x)$ and $g_{p, \pi}^{r}(x)$. Note that if $r \geq 1$ then $h_{p}^{r}\left(\sigma_{p}\right)=g_{p}^{r}\left(\sigma_{p+1}\right)=0$.

Lemma 1: The next equation holds.

$$
V_{n}=\sum_{m=0}^{n-1} \int_{\sigma_{m}}^{\sigma_{m+1}} g_{m}^{n-1-m}(x) e^{-s\left(x-\sigma_{m}\right)} V_{m}(x) d x .
$$

Proof: To prove the equation, we use integration by parts. Let us start from the following recursion form :

$$
V_{n}=\int_{0}^{\sigma_{n}} e^{-s \tau_{n} v}\left(\frac{x-\sigma_{n-1}}{\tau_{n}}\right) V_{n-1}(x) d x .
$$

From the definition of $v$,

$$
\begin{aligned}
V_{n}= & \int_{\sigma_{n-1}}^{\sigma_{n}} e^{-s\left(x-\sigma_{n}\right)} V_{n-1}(x) d x \\
& +\int_{0}^{\sigma_{n-1}} V_{n-1}(x) d x .
\end{aligned}
$$

It is expressed by

$$
\begin{aligned}
V_{n}= & \int_{\sigma_{n-1}}^{\sigma_{n}} g_{n-1}^{0}(x) e^{-s\left(x-\sigma_{n}\right)} V_{n-1}(x) d x \\
& +\int_{0}^{\sigma_{n-1}}\left(-g_{n-2}^{1}(x)\right)^{\prime} V_{n-1}(x) d x .
\end{aligned}
$$

By using integration by parts, the second term is changed to the following form :

$$
\int_{0}^{\sigma_{n-1}} g_{n-2}^{1}(x) e^{-s \tau_{n-2} v\left(\frac{x-\sigma_{n-2}}{\tau_{n-1}}\right)} V_{n-2}(x) d x,
$$

and the term is expressed by the following form from the definition of $v$ :

$$
\begin{aligned}
& \int_{\sigma_{n-2}}^{\sigma_{n-1}} g_{n-2}^{1}(x) e^{-s\left(x-\sigma_{n-2}\right)} V_{n-2}(x) d x \\
& \quad+\int_{0}^{\sigma_{n-2}} g_{n-2}^{1}(x) V_{n-2}(x) d x .
\end{aligned}
$$

By continuing these processes, the equation is obtained.

Lemma 2: For every $x \geq \sigma_{m}$, the next equation holds.

$$
V_{m}(x)=\sum_{r=0}^{m} e^{-s\left(\sigma_{m}-\sigma_{m}\right)} V_{m-r} h_{m}^{r}(x) .
$$

Proof: By definition, $V_{0}(x)=1$. For every $x \geq \sigma_{1}$,

$$
\begin{aligned}
V_{1}(x) & =V_{1}+\int_{\sigma_{1}}^{x} e^{-s \tau_{1}} V_{0}(t) d t \\
& =V_{1}+e^{-s \tau_{1}} V_{0} h_{1}^{1}(x) .
\end{aligned}
$$


For every $x \geq \sigma_{2}$,

$$
V_{2}(x)=V_{2}+\int_{\sigma_{2}}^{x} e^{-s \tau_{2}} V_{1}(t) d t
$$

and by substituting the former equation, it is expressed by

$$
\begin{aligned}
V_{2}(x)= & V_{2}+e^{-s\left(\sigma_{2}-\sigma_{1}\right)} V_{1} h_{2}^{1}(x) \\
& +e^{-s\left(\sigma_{2}-\sigma_{0}\right)} V_{0} h_{2}^{2}(x) .
\end{aligned}
$$

By continuing these processes, the proof is complet. ed.

Lemma 3 : The next equation holds.

$$
\begin{aligned}
& \frac{d}{d x} h_{k}^{k}\left(x+\sigma_{k}\right)=h_{k-1}^{k-1}\left(x+\sigma_{k}\right) \\
& =\sum_{r=0}^{k-1} h_{k-1-r}^{k-1-r}\left(\sigma_{k-r}\right) h_{k}^{r}\left(x+\sigma_{k}\right) .
\end{aligned}
$$

Proof: The first equality is obvious. To prove the second identity, we use the relations $h_{p}^{q}\left(\sigma_{p}\right)=0$ if $q$ $\geq 1$ and $h_{p}^{0}(x)=1$. By comparing both differential coefficients of the $i$-th order $i=0,1, \ldots, k-1$ at $x=$ $\sigma_{k-i}-\sigma_{k}$, the equality is proved.

Lemma 4 : The next equation holds.

$$
\begin{aligned}
& -\frac{d}{d x} g_{k}^{n-1-k}\left(x+\sigma_{k}\right)=g_{k+1}^{n \cdots 1 \cdots \cdot 1}\left(x+\sigma_{k}\right) \\
& =\sum_{m=k+1}^{n-1} g_{n-m+k}^{m-k-1}\left(\sigma_{n-m+k}\right) g_{k}^{n-1-m}\left(x+\sigma_{k}\right) .
\end{aligned}
$$

The proof is omitted because it is similar to lemma 3 .

Lemma 5 : The next equation holds.

$$
\begin{aligned}
& \sum_{\pi \in P_{n} m=1} \sum_{r=0}^{n-1} \sum_{r=0}^{m-1} g_{n-m+r, \pi}^{m+r}\left(\sigma_{n-m+r, \pi}\right) \\
& \int_{0}^{\tau_{\pi(r+1)}} e^{-s\left(x+\sigma_{r, \pi}\right)} g_{r, \pi}^{n-1-m}\left(x+\sigma_{r, \pi}\right) h_{r, \pi}^{r}\left(x+\sigma_{r, \pi}\right) d x \\
& =\sum_{m=1}^{n-1} \sum_{r=0}^{m-1} \sum_{\pi \in P_{n}} g_{0, \pi}^{m-r-1}(0) \\
& \int_{0}^{\tau_{\pi(m+1)}} e^{-s\left(x+\sigma_{m, \pi}-\sigma_{m-r, \pi}\right)} \\
& g_{m, \pi}^{n-1-m}\left(x+\sigma_{m, \pi}\right) h_{m, \pi}^{r}\left(x+\sigma_{m, \pi}\right) d x .
\end{aligned}
$$

Proof: We change the left side. It is clear that the first $\Sigma$ and other two $\Sigma$ 's are commutative. Since $r<m<n$, a permutation of the form

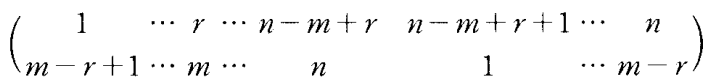

can be applied before the permutation $\pi$ is applied. As for the integral part, the permutation is firstly applied and a change of variable secondly applied to $g$ and $h$ by $y_{i}=t_{i}+\sigma_{m-r, \pi}$. As for $g$ of outside of integral, a change of variable is firstly applied by $y_{i}=t_{i}-\sigma_{n-m+r, \pi}$ and the permutation is secondly applied. It coincides with the right side.
Lemma 6 : The next equations hold for $j=1,2, \ldots$, $n$.

$$
\sum_{\pi \in P_{j}} g_{0, \pi}^{j-1}(0)=\sum_{\pi \in P_{j}^{2}} h_{j-1, \pi}^{i-1}\left(\sigma_{j, \pi}\right)
$$

Proof: If $j=1$, it is obvious. When $j \geq 2$, we change the left side. At first, a change of a variable is applied by $y_{i}=\sigma_{j, \pi}-t_{i}$. Secondly a permutation

$$
\left(\begin{array}{cccc}
1 & 2 & \cdots & j \\
j & j-1 & \cdots & 1
\end{array}\right)
$$

is applied before the permutation $\pi$ is applied. It coincides with the right side.

Lemma 7 : The distribution function is continuous. Proof : If $x_{1} \leq x_{2} \leq \cdots \leq x_{n}$, the next equation holds.

$$
\begin{gathered}
\frac{\partial}{\partial x_{i}} \sum_{i=1}^{n} \tau_{i} v\left(\frac{x_{i}-\sigma_{i-1}}{\tau_{i}}\right)=0 \\
\text { if } x_{i}<\sigma_{i-1} \text { or } x_{i}>\sigma_{i} .
\end{gathered}
$$

Considering the relation $0=\sigma_{0} \leq \sigma_{1} \leq \sigma_{2} \leq \cdots \leq \sigma_{n}$ and $x_{i} \in\left[0, \sigma_{n}\right]$, we can show that every partial differential coefficient can not be 0 simultaneously. $x_{1}>\sigma_{1}, x_{2}>\sigma_{2}, \cdots, x_{n-1}>\sigma_{n-1} \Rightarrow$ the partial differential coefficient of $x_{n}$ can not be 0 . This implies that $P\left(\left(x_{1}, x_{2}, \ldots, x_{n}\right): \sum_{i=1}^{n} \tau_{i} v\left(\frac{x_{i}-\sigma_{i-1}}{\tau_{i}}\right)=\beta\right)=0$. This means that the distribution function is continuous.

This lemma will be used to show that the equality after Laplace transform means the equality before the transform.

\subsubsection{Proof of Theorem 1}

Here we prove theorem 1.

Proof: From lemma 1 and lemma 2,

$$
\begin{aligned}
& V_{n}=\sum_{m=0}^{n-1} \sum_{r=0}^{m} V_{m-r} \int_{0}^{\tau_{m+1}} e^{-s\left(x+\sigma_{m}-\sigma_{m-r}\right)} \\
& g_{m}^{n-1-m}\left(x+\sigma_{m}\right) h_{m}^{r}\left(x+\sigma_{m}\right) d x .
\end{aligned}
$$

It can be divided into two parts as follows:

$$
\begin{aligned}
& \left.V_{n}=\sum_{m=1}^{n-1} \sum_{r=0}^{m-1} V_{m-r} \int_{0}^{\tau_{m+1}} e^{-s\left(x+\sigma_{m}\right.} \sigma_{m-r}\right) \\
& g_{m}^{n-1-m}\left(x+\sigma_{m}\right) h_{m}^{r}\left(x+\sigma_{m}\right) d x \\
& \quad+\sum_{k=0}^{n-1} \int_{0}^{\tau_{k+1}} e^{-s\left(x+\sigma_{k}\right)} \\
& g_{k}^{n-1-k}\left(x+\sigma_{k}\right) h_{k}^{k}\left(x+\sigma_{k}\right) d x .
\end{aligned}
$$

The second part is divided into three parts i.e. $k=$ $0,1 \leq k \leq n-2$ and $k=n-1$. Furthermore they are changed to the forms for the usage of integration by parts as follows:

$$
\begin{aligned}
& \int_{0}^{\tau_{1}}\left(\frac{e^{-s x}}{-s}\right)^{\prime} g_{0}^{n-1}(x) d x \\
& +\sum_{k=1}^{n-2} \int_{0}^{\tau_{k+1}}\left(\frac{e^{-s\left(x+\sigma_{k}\right)}}{-s}\right)^{\prime}
\end{aligned}
$$




$$
\begin{aligned}
& g_{k}^{n-1-k}\left(x+\sigma_{k}\right) h_{k}^{k}\left(x+\sigma_{k}\right) d x \\
& \quad+\int_{0}^{\tau_{n}}\left(\frac{e^{-s\left(x+\sigma_{n-1}\right)}}{-s}\right)^{\prime} h_{n-1}^{n-1}\left(x+\sigma_{n-1}\right) d x
\end{aligned}
$$

At first, integration by parts is done. By using the left equalities of lemma 3 and lemma 4 , and recombining the terms, they are changed to

$$
\begin{aligned}
& \frac{1}{S}\left\{g_{0}^{n-1}(0)-e^{-s \sigma_{n}} h_{n-1}^{n-1}\left(\sigma_{n}\right)\right\} \\
& \quad+\sum_{k=1}^{n-1} \frac{1}{s} \int_{0}^{\tau_{k+1}} e^{-s\left(x+\sigma_{k}\right)} \\
& g_{k}^{n-1-k}\left(x+\sigma_{k}\right) h_{k-1}^{k-1}\left(x+\sigma_{k}\right) d x \\
& \quad-\sum_{k=0}^{n-2} \frac{1}{s} \int_{0}^{\tau_{k+1}} e^{-s\left(x+\sigma_{k}\right)} \\
& g_{k+1}^{n-1-k-1}\left(x+\sigma_{k}\right) h_{k}^{k}\left(x+\sigma_{k}\right) d x .
\end{aligned}
$$

By substituting the right identities of lemma 3 and lemma 4 , and using a relation $\sum_{r=0}^{n-2} \sum_{m=r+1}^{n-1}=\sum_{m=1}^{n-1} \sum_{r=0}^{m-1}$ for the third therm,

$$
\begin{aligned}
& V_{n}=\frac{1}{s}\left\{g_{0}^{n-1}(0)-e^{-s \sigma_{n}} h_{n-1}^{n-1}\left(\sigma_{n}\right)\right\} \\
& \quad+\sum_{m=1}^{n-1} \sum_{r=0}^{m-1} V_{m-r} \int_{0}^{\tau_{m+1}} e^{-s\left(x+\sigma_{m}-\sigma_{m-r}\right)} \\
& \quad g_{m}^{n-1-m}\left(x+\sigma_{m}\right) h_{m}^{r}\left(x+\sigma_{m}\right) d x \\
& \quad+\sum_{m=1}^{n-1} \sum_{r=0}^{m-1} \frac{1}{s} e^{-s \sigma_{m}+} h_{m-r-1}^{m-r-1}\left(\sigma_{m-r}\right) \\
& \int_{0}^{\tau_{m+1}} e^{-s\left(x+\sigma_{m}-\sigma_{m-r}\right.} \\
& g_{m}^{n-1-m}\left(x+\sigma_{m}\right) h_{m}^{r}\left(x+\sigma_{m}\right) d x \\
& -\sum_{m=1}^{n-1} \sum_{r=0}^{m-1} \frac{1}{s} g_{n-m+r}^{m-r-1}\left(\sigma_{n-m+r}\right) \\
& \int_{0}^{\tau_{r+1}} e^{-s\left(x+\sigma_{r}\right)} \\
& g_{r}^{n-1-m}\left(x+\sigma_{r}\right) h_{r}^{r}\left(x+\sigma_{r}\right) d x
\end{aligned}
$$

holds.

Here we operate $\sum_{\pi \in P_{n}}$ to the both side of the above equation. Substitute the result of lemma 5 and replace $r$ by $j=m-r$. The following equation is deduced:

$$
\begin{aligned}
\sum_{\pi \in P_{n}}( & \left.V_{n, \pi}-\frac{1}{s} g_{0, \pi}^{n-1}(0)+\frac{1}{S} e^{-s \sigma_{n}} h_{n-1, \pi}^{n-1}\left(\sigma_{n}\right)\right) \\
= & \sum_{m-1}^{n-1} \sum_{j=1}^{m} \sum_{\pi \in P_{n}}\left[\left(\int_{0}^{\tau_{\pi(m+1)}} e^{-s\left(x+\sigma_{m, \pi}-\sigma_{, \pi, \pi}\right)}\right.\right. \\
& \left.g_{m, \pi}^{n-1-m}\left(x+\sigma_{m, \pi}\right) h_{m, \pi}^{m-j}\left(x+\sigma_{m, \pi}\right) d x\right) . \\
& \left.\left(V_{j, \pi}-\frac{1}{S} g_{0, \pi}^{j-1}(0)+\frac{1}{S} e^{-s \sigma_{j, \pi}} h_{j-1, \pi}^{j-1}\left(\sigma_{j, \pi}\right)\right)\right] .
\end{aligned}
$$

Here we use the fact that

$\int_{0}^{\left.\tau_{\pi i m+1}\right)} e^{-s\left(x+\sigma_{m, \pi}-\sigma_{, \pi, \pi}\right)} g_{m, \pi}^{n-1-m}\left(x+\sigma_{m, \pi}\right) h_{m, \pi}^{m-j}\left(x+\sigma_{m, \pi}\right) d x$ does not depend on $\tau_{\pi_{(1)}}, \tau_{\pi_{(2)}}, \ldots, \tau_{\left.\pi_{(j)}\right)}$. This integral part is denoted by $z\left(\tau_{\pi(j+1)}, \tau_{\pi(j+2)}, \ldots, \tau_{\pi(n)}\right)$.
From lemma 6, we can set

$$
\begin{aligned}
a_{j, \pi}= & \sum_{\rho \in P_{j}} g_{0, \pi}^{j-1}(0)=\sum_{\rho \in P_{j}} h_{j-1, \pi}^{j-1}\left(\sigma_{j, \pi}\right), \\
& j=1,2, \ldots, n,
\end{aligned}
$$

where $\pi \circ \rho$ is the composite permutation, and the permutation $\rho$ is assumed to be

$$
\left(\begin{array}{cccccc}
1 & \cdots & j & j+1 & \cdots & n \\
\rho(1) & \cdots & \rho(j) & j+1 & \cdots & n
\end{array}\right) .
$$

If $\pi$ is the identity permutation, it is simply denoted by $a_{j}$.

Thus the equation is changed to the following recursion form :

$$
\begin{aligned}
& \sum_{\pi \in P_{n}} V_{n, \pi}-\frac{a_{n}}{s}\left(1-e^{-s \sigma_{n}}\right)= \\
& \quad \sum_{m=1}^{n-1} \sum_{j=1}^{m} \frac{1}{j !} \sum_{\pi \in P_{n}}\left(z\left(\tau_{n(j+1)}, \tau_{n(j+2)}, \ldots, \tau_{n(n)}\right) \cdot\right. \\
& \left.\quad\left(\sum_{\rho \in P_{j}} V_{j, \pi}-\frac{a_{j, \pi}}{s}\left(1-e^{-s \sigma_{, j}}\right)\right)\right) .
\end{aligned}
$$

Since $V_{1, \pi}=\frac{1}{S}\left(1-e^{-s \sigma_{1, \pi}}\right)$ and $a_{1, \pi}=g_{0, \pi}^{0}(0)=$ $h_{0, \pi}^{0}\left(\sigma_{1, \pi}\right)=1$ are valid, by using mathematical induction the following equation holds :

$$
\sum_{\pi \in P_{n}} V_{n, \pi}=\frac{a_{n}}{s}\left(1-e^{-s \sigma_{n}}\right) .
$$

Here we use the final value theorem of Laplace transform.

$$
F(\infty)=\lim _{s \rightarrow 0} s V(s) .
$$

$\lim _{s \rightarrow 0} s V(s)=\lim _{s \rightarrow 0} \sum_{\pi \in P_{n}} V_{n, \pi}=a_{n} \sigma_{n}$ holds. By comparing this with the fact $F(\infty)=\left(\sigma_{n}\right)^{n}$, the following equation is deduced :

$$
a_{n}=\left(\sigma_{n}\right)^{n-1} \text {. }
$$

Thus the following equation holds :

$$
\sum_{\pi \in P_{n}} V_{n, \pi}=\frac{1}{s}\left(\sigma_{n}\right)^{n-1}\left(1-e^{-s \sigma_{n}}\right) .
$$

Finally, from lemma 7 , by using inversion for Laplace transform, we can conclude that if $\sigma_{n}=1$ then

$$
F(\beta)=v(\beta)
$$

holds.

\section{Conclusions}

A fuzzy integral named opposite Sugeno integral was investigated as an averaging operator. We focused on a variance diminishing property after averaging. The discussion was limited in the case that each original distribution of $n$-variables is assumed to be uniform distribution independently. 
Our concern was of course the distribution function after integration. In general, the distribution function depends on fuzzy measure.

We precisely studied the variance of distribution functions in the case of two variables. For every fuzzy measure, the variances did not exceed $1 / 12$ which was variance of the original distribution. Opposite Sugeno integral therefore has a variance diminishing property. If fuzzy measure is unit mass i.e. $\tau_{1}=1, \tau_{2}=0$ or $\tau_{1}=0, \tau_{2}=1$, it is trivial that the distribution after integration is equal to the original one. After all, in opposite Sugeno integral, the same distribution appeared with more relaxed condition that fuzzy measure is additive.

By comparing with Sugeno integral, the fact is a typical characteristic of opposite Sugeno integral, and we have proved it in general i.e. in the case of $n$-variables.

What will happen if the original distribution is asuumed to be a bell shape like the normal distribution? This is one of the future works, but we can imagine that the variance after opposite Sugeno integration will be smaller than the original one and it will be larger than the variance after ordinary averaging.

\section{References}

[1] H.Imaoka, "On a subjective evaluation model by a generalized fuzzy integral," Int. J. of Uncertuinty, Fuzziness and Knowledge-Based Systems, 5(1997), $517-529$.

[2] M.Sugeno, "Theory of fuzzy integral and its applications," Doct. Thesis, Tokyo Institute of Technology (1974).

$$
\text { （1999年5月25口 受付） }
$$

[Contact Address]

Haruki IMAOKA

Faculty of Human Life and Environment,

Nara Women's University

Kitauoya Nishimachi, Nara 630-8506 JAPAN

TEL : + 81-742-203465

FAX : + 81-742-203499

E-mail : imaoka@cc.nara-wu.ac.jp

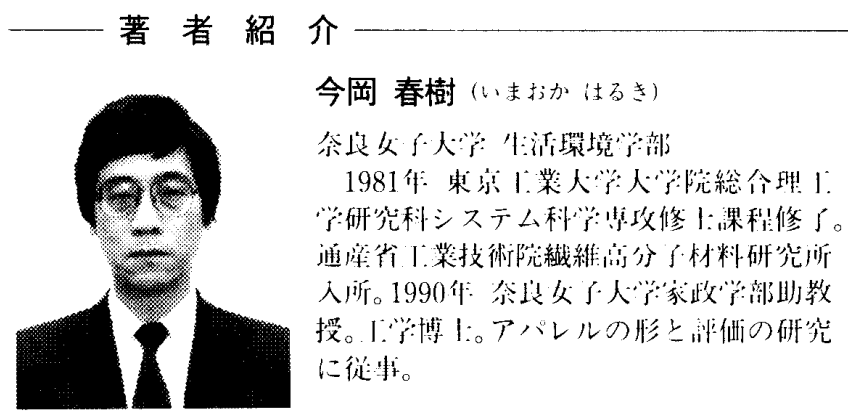

一様分布入カべクトルの逆菅野積分値の分布は一様

今岡 春樹

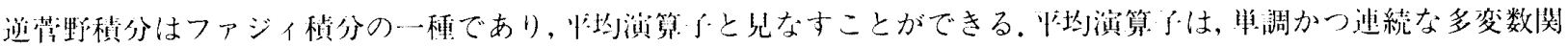

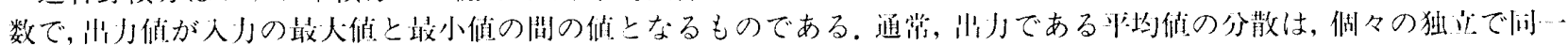

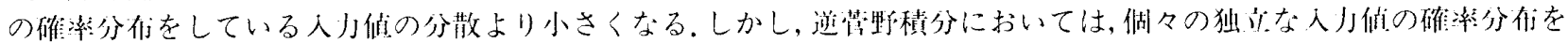

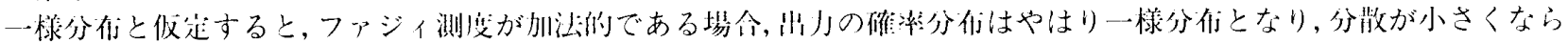
ない。

キーワード：ファジィ棈分，逆菅野植分，一椂分倠

\author{
[開い命わせ先]

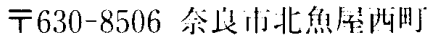

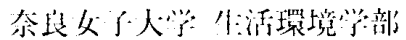 \\ 今湔焦樹 \\ TEL : +81-742-203465 \\ FAX : +81-742-203499 \\ Email :imaoka@cc.nara-wu.ac.jp
}

